ESAIM: PROCEEDINGS AND SURVEYS, September 2014, Vol. 45, p. 8-17

J.-S. Dhersin, Editor

\title{
CONSERVATION LAWS WITH LOCAL FLUX CONSTRAINTS ARISING IN TRAFFIC FLOW MODELING*
}

\author{
PAOLA GOATIN ${ }^{1}$
}

\begin{abstract}
Specific traffic flow conditions, such as the presence of toll gates, construction sites or moving bottlenecks caused by slow moving vehicles, can be realistically modeled by conservation laws with local unilateral constraints on the flux. We give an overview of the related analytical and numerical results.

Résumé. Certaines spécificités de la circulation routière, comme la présence de stations de péage, de sites de travaux ou encore de véhicules lents, peuvent être modélisées par des lois de conservation avec une contrainte unilatérale sur le flux. L'article donne une vue d'ensemble des résultats analytiques et numériques correspondant.
\end{abstract}

\section{INTRODUCTION}

Macroscopic traffic flow models make use of partial differential equations derived from fluid dynamics to describe the spatio-temporal evolution of macroscopic quantities such as the traffic density $\rho$ and the mean velocity $v$. Starting from the basic assumption that the number of cars on an infinite road is conserved, one easily recover a mass conservation law in one space dimension, in the form

$$
\partial_{t} \rho+\partial_{x}(\rho v)=0, \quad x \in \mathbb{R}, t>0
$$

where $\rho=\rho(t, x) \in[0, R]$ and $v=v(t, x) \in\left[0, V_{\max }\right], R$ and $V_{\max }$ denoting respectively the maximal density and the maximal speed on the considered road.

First order traffic flow models consist in equation (1) only, taking the mean velocity as a non-increasing function of the density. In this survey, we will assume

$$
v=v(\rho), \quad \text { such that } v(0)=V_{\max } \text { and } v(R)=0 .
$$

Therefore, equation (1) can be rewritten as

$$
\partial_{t} \rho+\partial_{x} f(\rho)=0, \quad x \in \mathbb{R}, t \geq 0,
$$

where the flux function $f:[0, R] \rightarrow \mathbb{R}^{+}$is defined by $f(\rho)=\rho v(\rho)$. Dealing with traffic flow applications, it is not restrictive to assume that $f$ is Lipschitz continuous and satisfies

$$
f(\rho) \geq 0, \quad f(0)=f(R)=0, \quad f^{\prime}\left(\rho_{c}\right)\left(\rho_{c}-\rho\right)>0 \text { a.e. } \rho \in[0, R]
$$

\footnotetext{
* The author was partially supported by the European Research Council under the European Union's Seventh Framework Program (FP/2007-2013) / ERC Grant Agreement n. 257661.

${ }^{1}$ INRIA Sophia Antipolis - Méditerranée, 2004, route des Lucioles - BP 93, 06902 Sophia Antipolis Cedex, France
}

(C) EDP Sciences, SMAI 2014 
where $\left.\rho_{c} \in\right] 0, R[$ denotes the point of maximum of the flux, see Figure 1 . In the following we will use the notation

$$
f_{\max }=f\left(\rho_{c}\right) .
$$
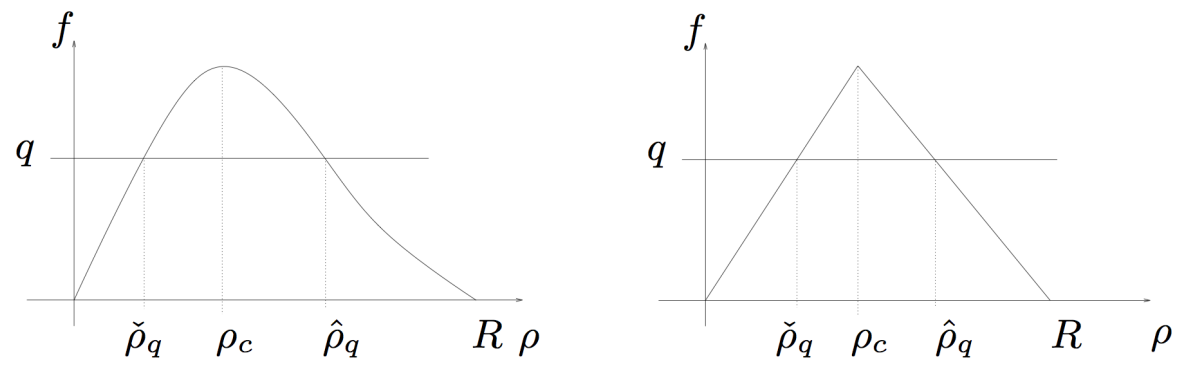

FiguRE 1. Examples of fundamental diagrams considered here.

Specific traffic conditions, such as the presence of a toll gate of other situations that hinder the flow of vehicles, can be modeled by imposing an upper bound on the flux at the location of the constraint $x=\bar{x}$

$$
f(\rho(t, \bar{x})) \leq q(t)
$$

Observe that the constraint is in general time-dependent: for example, it depends on the number of open gates at a toll station.

\section{Constrained Entropy Weak solutions}

Let us consider the following constrained Cauchy problem

$$
\begin{cases}\partial_{t} \rho+\partial_{x} f(\rho)=0, & x \in \mathbb{R}, t>0 \\ \rho(0, x)=\rho_{0}(x), & x \in \mathbb{R} \\ f(\rho(t, 0)) \leq q(t), & t>0\end{cases}
$$

where $\rho_{0} \in \mathbf{L}^{\infty}(\mathbb{R} ;[0, R])$ and $q \in \mathbf{L}^{\infty}\left(\mathbb{R}^{+} ;\left[0, f_{\text {max }}\right]\right)$. Problem (5) can be seen as the singular limit of a classical Cauchy problem with discontinuous flux. Fix $\varepsilon>0$ and consider the problem

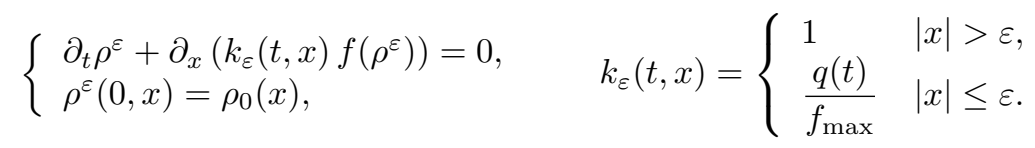

(We refer the interested reader to [5] for a rigorous justification of the limiting procedure.) Problem (6) fit the framework of conservation laws with discontinuous-in-space flux functions. In particular [12, Theorem 4.5, 5.5 and 6.5] apply, and the corresponding entropy condition is given by

$$
\begin{aligned}
\int_{0}^{+\infty} \int_{\mathbb{R}}\left(\left|\rho^{\varepsilon}(t, x)-\kappa\right| \partial_{t}+\Phi\left(\rho^{\varepsilon}(t, x), \kappa\right) \partial_{x}\right) \phi(t, x) d x & d t+\int_{\mathbb{R}}\left|\rho_{0}(x)-\kappa\right| \phi(0, x) d x \\
& +\int_{0}^{+\infty}\left(1-\frac{q(t)}{f_{\max }}\right) f(\kappa)(\phi(t,-\varepsilon)+\phi(t, \varepsilon)) d t \geq 0
\end{aligned}
$$


for all $\kappa \in[0, R]$ and $\phi \in C_{c}^{1}\left(\mathbb{R}^{+} \times \mathbb{R} ; \mathbb{R}^{+}\right)$, where we have noted $\Phi(a, b)=\operatorname{sgn}(a-b)(f(a)-f(b))$. Remark that $\rho^{\varepsilon}$ satisfy the constraint $f\left(\rho^{\varepsilon}(t, x)\right) \leq q(t)$ for a. e. $|x|<\varepsilon$.

Therefore, we give the following definition for (5):

Definition 1.1. A function $\rho \in \mathbf{L}^{\infty}\left(\mathbb{R}^{+} \times \mathbb{R} ;[0, R]\right)$ is an entropy weak solution of (5) if

(i) entropy inequalities are satisfied: for all $\phi \in C_{c}^{1}\left(\mathbb{R}^{+} \times \mathbb{R} ; \mathbb{R}^{+}\right)$and every $\kappa \in[0, R]$,

$$
\begin{aligned}
& \int_{0}^{+\infty} \int_{\mathbb{R}}\left(|\rho(t, x)-\kappa| \partial_{t}+\Phi(\rho(t, x), \kappa) \partial_{x}\right) \phi(t, x) d x d t+\int_{\mathbb{R}}\left|\rho_{0}(x)-\kappa\right| \phi(0, x) d x \\
&+2 \int_{0}^{+\infty}\left(1-\frac{q(t)}{f_{\max }}\right) f(\kappa) \phi(t, 0) d t \geq 0
\end{aligned}
$$

(ii) the constraint is satisfied:

$$
f\left(\left(\gamma^{l} \rho\right)(t)\right)=f\left(\left(\gamma^{r} \rho\right)(t)\right) \leq q(t) \quad \text { a. e. } t>0,
$$

where $\gamma^{l, r}$ are the left and right strong trace operators at $\{x=0\}$.

It is worth noting that the traces $\gamma^{l, r} u$ in (8) do exist $[14,16]$. Indeed, we are dealing with a flux function such that the measure of the set $\left\{s \in[0,1], f^{\prime}(s)=0\right\}$ is zero (by assumption (3)).

The above definition was first proposed in [5]. In order to facilitate dealing with limiting procedures of approximate solutions, and to dispose of a more compact formulation, we can get rid of the explicit condition (8) using an equivalent definition given in [2]. We introduce the set of admissible traces $\mathcal{G}(q)=\mathcal{G}_{1}(q) \cup \mathcal{G}_{2}(q) \cup \mathcal{G}_{3}(q)$ defined by

- $\mathcal{G}_{1}(q)=\left\{\left(c_{l}, c_{r}\right) \in[0, R]^{2} ; c_{l}>c_{r}, f\left(c_{l}\right)=f\left(c_{r}\right)=q\right\}$,

- $\mathcal{G}_{2}(q)=\left\{(c, c) \in[0, R]^{2} ; f(c) \leq q\right\}$,

- $\mathcal{G}_{3}(q)=\left\{\left(c_{l}, c_{r}\right) \in[0, R]^{2} ; c_{l}<c_{r}, f\left(c_{l}\right)=f\left(c_{r}\right) \leq q\right\}$.

Remark that, by $(3), \mathcal{G}_{1}(q)=\left\{\left(\hat{\rho}_{q}, \check{\rho}_{q}\right)\right\}$ is a singleton, uniquely defined by the conditions

$$
f\left(\hat{\rho}_{q}\right)=f\left(\check{\rho}_{q}\right)=q, \quad \hat{\rho}_{q} \geq \check{\rho}_{q},
$$

see Fig. 1.

Definition 1.2. A function $\rho \in \mathbf{L}^{\infty}\left(\mathbb{R}^{+} \times \mathbb{R} ;[0, R]\right)$ is an entropy weak solution of (5) if there exists $M>0$ such that for all $\left(c_{l}, c_{r}\right) \in[0, R]^{2}$ and all test functions $\phi \in C_{c}^{1}\left(\mathbb{R}^{+} \times \mathbb{R} ; \mathbb{R}^{+}\right)$,

$$
\begin{aligned}
\int_{0}^{+\infty} \int_{\mathbb{R}}\left(|\rho(t, x)-c(x)| \partial_{t}+\Phi(\rho(t, x), c(x)) \partial_{x}\right) \phi(t, x) d x d t & +\int_{\mathbb{R}}\left|\rho_{0}(x)-c(x)\right| \phi(0, x) d x \\
& +M \int_{0}^{+\infty} \operatorname{dist}\left(\left(c_{l}, c_{r}\right), \mathcal{G}(q(t))\right) \phi(t, 0) d t \geq 0
\end{aligned}
$$

where $c(x)$ is the piecewise constant function given by

$$
c(x):= \begin{cases}c_{l} & \text { if } x<0, \\ c_{r} & \text { if } x>0,\end{cases}
$$

with $\left(c_{l}, c_{r}\right) \in[0, R]^{2}$, and dist refers to a distance function on $\mathbb{R}^{2}$.

The equivalence of Definitions 1.1 and 1.2 is proved in [2, Proposition 2.6].

Remark 1.3. The above definitions select the maximal solution, since all non-classical stationary discontinuities at $x=0$ between two states $\rho_{2}$ and $\rho_{1}$ with $\rho_{1}<\rho_{2}$ and $f\left(\rho_{1}\right)=f\left(\rho_{2}\right)<q(t)$ are ruled out by (7) (equivalently, $(9))$. 


\section{WELL-POSEDNESS RESULTS}

\subsection{The Constrained Riemann Solver}

In this section, we consider the constrained Cauchy problem with Riemann type initial datum and constant constraint

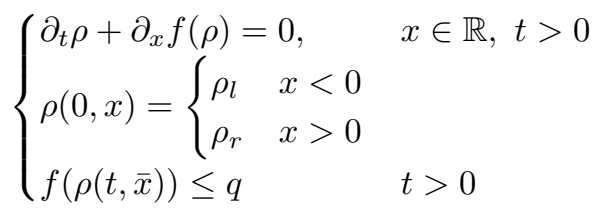

where $q \in\left[0, f_{\max }\right]$ and $\rho_{l}, \rho_{r} \in[0, R]$ are constants.

The self-similar solution to (10) can be described using the notion of standard Riemann solver, see [5]. We denote by $\mathcal{R}$ the standard Riemann solver for $(2)$, i.e. $(t, x) \mapsto \mathcal{R}\left(\rho^{l}, \rho^{r}\right)(x / t)$ is the standard weak entropy solution of (2), corresponding to the Riemann initial datum

$$
\rho(0, x)= \begin{cases}\rho_{l}, & x<0 \\ \rho_{r}, & x>0\end{cases}
$$

The following definition provides an entropy weak solution to (10) in the sense of Definitions 1.1, 1.2.

Definition 2.1. The Riemann solver $\mathcal{R}^{q}:\left(\rho_{l}, \rho_{r}\right) \mapsto \mathcal{R}^{q}\left(\rho_{l}, \rho_{r}\right)$ for $(10)$ is defined as follows.

- If $\left.f\left(\mathcal{R}\left(\rho_{l}, \rho_{r}\right)\right)(0)\right) \leq q$, then $\mathcal{R}^{q}\left(\rho_{l}, \rho_{r}\right)=\mathcal{R}\left(\rho_{l}, \rho_{r}\right)$.

- Otherwise, $\mathcal{R}^{q}\left(\rho_{l}, \rho_{r}\right)(\lambda)= \begin{cases}\mathcal{R}\left(\rho_{l}, \hat{\rho}_{q}\right)(\lambda) & \text { if } \lambda<0, \\ \mathcal{R}\left(\check{\rho}_{q}, \rho_{r}\right)(\lambda) & \text { if } \lambda>0 .\end{cases}$

Remark that, when the constraint is enforced, a stationary discontinuity violating the Lax entropy condition arises at $x=0$. For further properties of the Riemann solver defined above, we address the reader to [5, Proposition 2.3].

\subsection{The Constrained Cauchy Problem}

The standard approach to prove existence of solutions for conservation laws is to construct a sequence of approximate solutions which has uniformly bounded total variation. Then Helly's theorem guarantees the existence of a subsequence converging in $\mathbf{L}^{\mathbf{1}}$ towards a function that is showed to solve the equations.

In the present case (5), the presence of the constraint (4) may cause a sharp increase of TV $(\rho(t, \cdot))$. This is easily seen by taking the constant initial datum $\rho_{0}(x)=\rho_{c}$ and the constraint $q(t) \equiv f_{\max } / 2$. At time $t=0$, two shocks arise from $x=0$ and the total variation jumps from $\operatorname{TV}\left(\rho_{0}\right)=0$ to $\operatorname{TV}(\rho(t, \cdot))=2(\hat{\rho}-\check{\rho})$, where $\check{\rho}<\hat{\rho}$ and $f(\hat{\rho})=f(\check{\rho})=f_{\max } / 2$.

To overcome this difficulty, in [5] we introduced the nonlinear mapping

$$
\Psi(\rho)=\operatorname{sgn}\left(\rho-\rho_{c}\right)\left(f\left(\rho_{c}\right)-f(\rho)\right)
$$

and look for a bound on the total variation of $\Psi \circ \rho$. In fact, $\Psi$ is one-to-one, but possibly singular at $\rho=\rho_{c}$. Indeed, it is immediate to see that if $\rho \in \mathrm{BV}(\mathbb{R} ; \mathbb{R})$, then $\operatorname{TV}(\Psi \circ \rho) \leq\left\|f^{\prime}\right\|_{C^{0}} \cdot \operatorname{TV}(\rho)$, while $\operatorname{TV}(\rho)$ may well be infinite with $\Psi(\rho)$ finite, as in the case of $f(\rho)=\rho(1-\rho), \rho_{c}=1 / 2$ and $\rho=\sum_{n=3}^{+\infty} \frac{1}{n} \chi_{\left[\frac{1}{2}+\frac{2 n+1}{2 n(n+1)}, \frac{1}{2}+\frac{1}{n}[\right.}$.

The first well posedness result, stated in a BV framework, was proved in [5] and referred to the entropy condition given in Definition 1.1. 
Theorem 2.2. Assume that $\rho_{0} \in \mathcal{D}:=\left\{\rho \in \mathbf{L}^{\mathbf{1}}(\mathbb{R} ;[0, R]): \Psi(\rho) \in \mathrm{BV}(\mathbb{R} ; \mathbb{R})\right\}$ and $q \in \mathrm{BV}\left(\mathbb{R}^{+},\left[0, f_{\max }\right]\right)$. Then there exists a unique entropy weak solution $\rho \in \mathrm{BV}\left(\mathbb{R}^{+} \times \mathbb{R} ;[0, R]\right)$ to problem (5). Moreover, given two initial data $\rho_{0}^{1}, \rho_{0}^{2} \in \mathcal{D}$ such that $\left(\rho_{0}^{1}-\rho_{0}^{2}\right) \in \mathbf{L}^{\mathbf{1}}(\mathbb{R} ; \mathbb{R})$, the corresponding entropy weak solutions $\rho^{1}, \rho^{2}$ satisfy the following $\mathbf{L}^{\mathbf{1}}$-stability property: for a.e. $t>0$

$$
\left\|\rho^{1}(t, \cdot)-\rho^{2}(t, \cdot)\right\|_{\mathbf{L}^{1}(\mathbb{R} ; \mathbb{R})} \leq\left\|\rho_{0}^{1}-\rho_{0}^{2}\right\|_{\mathbf{L}^{1}(\mathbb{R} ; \mathbb{R})} .
$$

In [5], existence is proved using the wave-front tracking approximation technique and the functional (11). Note that, in the case of wave-front tracking approximations $\rho^{\nu}$, the traces $\gamma^{l, r} f(\rho)$ of the limit of $f\left(\rho^{\nu}\right)$ are the limits of the traces $\gamma^{l, r} f\left(\rho^{\nu}\right)$, respectively, thanks to the Green-Gauss formula. In fact, the constraint (8) is equivalent to the inequality

$$
\int_{0}^{+\infty} q(t) \xi(t) d t \geq \int_{0}^{+\infty} f\left(\left(\gamma^{l, r} \rho\right)(t)\right) \xi(t) d t= \pm \int_{0}^{+\infty} \int_{\mathbb{R}^{ \pm}}\left(\rho(t, x) \partial_{t}+f(\rho(t, x)) \partial_{x}\right) \xi(t) \phi(x) d x d t
$$

for all $\xi \in C_{c}^{\infty}\left(\mathbb{R}^{+} ; \mathbb{R}^{+}\right)$and some given $\phi \in C_{c}^{\infty}\left(\mathbb{R} ; \mathbb{R}^{+}\right), \phi(0)=1$. Hence (8) in Definition 1.1(ii) is verified. Uniqueness is obtained using the Kruzhkov doubling of variables technique ( [13]) on each side of the constraint location; the inequality (8) for the strong traces of $\rho$ is used to obtain the right inequalities across the interface $\{x=0\}$.

Theorem 2.2 has been generalized to initial-boundary value problems with application to traffic management in $[6]$.

Well-posedness has then been proved for $\mathbf{L}^{\infty}$ data in [2], relying on the formulation of the entropy condition given in Definition 1.2 and the following stability estimate:

Proposition 2.3. Assume that $q^{1}, q^{2} \in \mathbf{L}^{\infty}\left(\mathbb{R}^{+} ;\left[0, f_{\max }\right]\right)$, and $\rho_{0}^{1}, \rho_{0}^{2} \in \mathbf{L}^{\infty}(\mathbb{R},[0, R])$ such that $\left(\rho_{0}^{1}-\rho_{0}^{2}\right) \in$ $\mathbf{L}^{\mathbf{1}}(\mathbb{R} ; \mathbb{R})$. Assume that $\rho^{1}, \rho^{2}$ are entropy weak solutions of problem (5) corresponding to the initial data $\rho_{0}^{1}, \rho_{0}^{2}$ and the constraints $q^{1}, q^{2}$, respectively. Then, for a.e. $t>0$, we have

$$
\left\|\rho^{1}(t, \cdot)-\rho^{2}(t, \cdot)\right\|_{\mathbf{L}^{1}(\mathbb{R} ; \mathbb{R})} \leq\left\|\rho_{0}^{1}-\rho_{0}^{2}\right\|_{\mathbf{L}^{1}(\mathbb{R} ; \mathbb{R})}+2\left\|q^{1}-q^{2}\right\|_{\mathbf{L}^{1}([0, t] ; \mathbb{R})} .
$$

Combining the above stability estimate with the existence result given in Theorem 2.2, and using a truncation and density argument, we get well-posedness in $\mathbf{L}^{\infty}$ :

Theorem 2.4. For any $\rho_{0} \in \mathbf{L}^{\infty}(\mathbb{R} ;[0, R])$ and $q \in \mathbf{L}^{\infty}\left(\mathbb{R}^{+} ;\left[0, f_{\max }\right]\right)$, there exists a unique entropy weak solution to problem (5).

The proof can be found in [2]. We remark here that the entropy formulation (9) is essential to pass to the limit in the regularization by convolution of the constraint function $q$.

\section{Constrained finite volume schemes}

In this section, we explain how to extend classical finite volume schemes to the constrained problem (5). Let a space step $\Delta x$ and a time step $\Delta t$ be given, both assumed to be constant for sake of simplicity. We define the mesh interfaces $x_{j+1 / 2}=j \Delta x$ for $j \in \mathbb{Z}$ and the intermediate times $t^{n}=n \Delta t$ for $n \in \mathbb{N}$. The finite volume approximation of the initial datum $\rho_{0}$ is

$$
\rho_{j}^{0}=\frac{1}{\Delta x} \int_{x_{j-1 / 2}}^{x_{j+1 / 2}} \rho_{0}(x) d x, \quad j \in \mathbb{Z},
$$

and the constraint value at each time step is approximated by

$$
q^{n}=\int_{t^{n}}^{t^{n+1}} q(s) d s, \quad n \in \mathbb{N}
$$


We aim at defining a sequence $\left(\rho_{j}^{n}\right)_{j \in \mathbb{Z}, n \in \mathbb{N}}$ which approximates the solution $\rho$ of $(5)$ in the sense

$$
\rho_{j}^{n} \approx \frac{1}{\Delta x} \int_{x_{j-1 / 2}}^{x_{j+1 / 2}} \rho\left(t^{n}, x\right) d x, \quad j \in \mathbb{Z}, n>0 .
$$

For any classical numerical flux $h(u, v)$ such that

(1) $h$ is Lipschitz continuous, with Lipschitz constant $L$,

(2) $h(u, u)=f(u)$ for any $u \in[0, R]$ (consistency),

(3) $h$ is non-decreasing w.r.t. $u$ and non-increasing w.r.t. $v$ (monotonicity),

we define

$$
g\left(\rho_{j}^{n}, \rho_{j+1}^{n}, q^{n}\right)= \begin{cases}\min \left\{h\left(\rho_{j}^{n}, \rho_{j+1}^{n}\right), q^{n}\right\}, & \text { if } j=0, \\ h\left(\rho_{j}^{n}, \rho_{j+1}^{n}\right) & \text { if } j \neq 0 .\end{cases}
$$

Then the updating formula is given by

$$
\rho_{j}^{n+1}=\rho_{j}^{n}-\frac{\Delta t}{\Delta x}\left(g\left(\rho_{j}^{n}, \rho_{j+1}^{n}, q^{n}\right)-g\left(\rho_{j-1}^{n}, \rho_{j}^{n}, q^{n}\right)\right), \quad j \in \mathbb{Z}, n \in N
$$

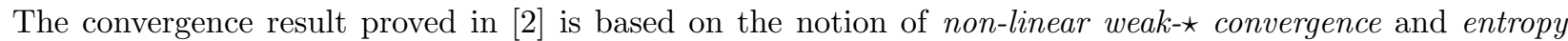
process solutions, see [10]. This approach is useful for studying convergence of finite volume schemes, because it only requires an $\mathbf{L}^{\infty}$ estimates on the sequence of discrete solutions (for $\mathbf{L}^{\infty}$ weak- $\star$ compactness) and a "weak BV" estimate (for consistency). As such, it can be used for treating problems where strong compactness properties are lacking. In the present case, the numerical scheme (14), (15) satisfies the following estimates.

Proposition 3.1. ( $\mathbf{L}^{\infty}$ estimate) Assume that $\rho_{0} \in \mathbf{L}^{\infty}(\mathbb{R} ;[0, R])$. Then, under the CFL condition

$$
\Delta t \leq \frac{\Delta x}{2 L}
$$

the numerical scheme (14), (15) is monotone and the finite volume approximation (15) satisfies

$$
0 \leq \rho_{j}^{n} \leq R, \quad \forall j \in \mathbb{Z}, \forall n \in \mathbb{N}
$$

Proposition 3.2. (Weak-BV estimate) Let $\xi \in(0,1)$ and $T>\Delta t$ and $M>\Delta x$ be two positive constants and denote by $I_{0}, I_{1}$ and $N$ the indices such that $\left.\left.\left.\left.-M \in\right] x_{I_{0}-1 / 2}, x_{I_{0}+1 / 2}\right], M \in\right] x_{I_{1}-1 / 2}, x_{I_{1}+1 / 2}\right]$ and $T \in$ ] $N \Delta t,(N+1) \Delta t]$. Then, if the time step $\Delta t$ satisfies the CFL condition

$$
\Delta t \leq(1-\xi) \frac{\Delta x}{2 L}
$$

there exists a positive constant $C$ only depending on $T, M, \xi$, $f$ and $\rho_{0}$ such that

$$
\Delta t \sum_{n=0}^{N} \sum_{\substack{i=I_{0} \\ i \neq 0,1}}^{I_{1}}\left(\max _{(p, q) \in \mathbb{I}\left(\rho_{i}^{n}, \rho_{i+1}^{n}\right)}|h(p, q)-f(p)|+\max _{(p, q) \in \mathbb{I}\left(\rho_{i}^{n}, \rho_{i+1}^{n}\right)}|h(p, q)-f(q)|\right) \leq C \sqrt{\Delta x}
$$

where $\mathbb{I}(a, b)=\{(p, q) \in[\min \{a, b\}, \max \{a, b\}],(q-p)(b-a) \geq 0\}$.

The above properties guarantee weak- $\star$ convergence. In order to show that the limit is an entropy process solution, one needs discrete entropy inequalities. 
Proposition 3.3. Let $\kappa_{j}=c_{l}$ for $j \leq 0$ and $\kappa_{j}=c_{r}$ for $j>0$, where $\left(c_{l}, c_{r}\right) \in[0, R]^{2}$. Then, the numerical scheme (14), (15) fulfills the following inequalities:

$$
\left|\rho_{j}^{n+1}-\kappa_{j}\right|-\left|\rho_{j}^{n}-\kappa_{j}\right|+\frac{\Delta t}{\Delta x}\left(G_{j+1 / 2}^{n}-G_{j-1 / 2}^{n}\right)-\frac{\Delta t}{\Delta x}\left|H_{j}^{n}\right| \leq 0
$$

where

$$
\begin{aligned}
G_{j+1 / 2}^{n} & =g\left(\max \left\{\rho_{j}^{n}, \kappa_{j}\right\}, \max \left\{\rho_{j+1}^{n}, \kappa_{j+1}\right\}, q^{n}\right)-g\left(\min \left\{\rho_{j}^{n}, \kappa_{j}\right\}, \min \left\{\rho_{j+1}^{n}, \kappa_{j+1}\right\}, q^{n}\right), \\
H_{j}^{n} & = \begin{cases}\min \left\{h\left(\kappa_{j}, \kappa_{j+1}\right), q^{n}\right\}-\min \left\{h\left(\kappa_{j-1 / 2}, \kappa_{j}\right), q^{n}\right\}, & \text { if } j=0, \\
h\left(\kappa_{j}, \kappa_{j+1}\right)-h\left(\kappa_{j-1 / 2}, \kappa_{j}\right), & \text { if } j \neq 0,\end{cases}
\end{aligned}
$$

for all $j \in \mathbb{Z}$ and $n \in \mathbb{N}$.

By uniqueness of the entropy process solution, see [2, Proposition 3.3], the numerical approximations converge indeed towards the entropy weak solution in the sense of Definitions 1.1, 1.2.

Theorem 3.4. Under the CFL condition (16), the finite volume scheme (14), (15) converges in $\mathbf{L}^{\mathbf{p}}$ loc $\left(\mathbb{R}^{+} \times \mathbb{R} ; \mathbb{R}\right)$ for any $1 \leq p<+\infty$ to the unique entropy weak solution of (5).

\section{FURTHER RESUlts}

The results described in the previous sections have then been extended to other models arising in traffic flow modeling. We list here the main achievements.

\subsection{General fluxes}

The pedestrian flow model introduced in [7] displays a non concave flux function. Therefore, in [4] the flux function is assumed to comply with the following hypotheses:

(H1) $f \in \mathbf{W}^{1, \infty}\left([0, R] ;\left[0,+\infty[)\right.\right.$ and piecewise $C^{1}$,

(H2) $f(\rho) \geq 0, f(0)=f(R)=0$,

(H3) there exists a finite set of points $\left\{\rho_{1}, \ldots, \rho_{N}\right\} \subset[0, R], N \geq 1$, which are strict local minima or maxima of $f$ i.e. $f(\rho)-f\left(\rho_{i}\right)<0$ (or $>0$ ) for $\rho$ in a neighborhood of $\rho_{i}, i=1, \ldots, N$, and $f^{\prime}(\rho \pm) \neq 0$ for all $\rho \in[0, R] \backslash\left\{\rho_{1}, \ldots, \rho_{N}\right\}$.

In this setting, the entropy condition needs to be slightly modified. Indeed, Definition 1.1 turn in the following

Definition 4.1. A function $\rho \in \mathbf{L}^{\infty}\left(\mathbb{R}^{+} \times \mathbb{R} ;[0, R]\right)$ is a weak entropy solution of (5), with $f$ satisfying (H1)-(H3), if

(i) it satisfies the following entropy inequalities: for every $\phi \in C_{c}^{1}\left(\mathbb{R}^{+} \times \mathbb{R} ; \mathbb{R}^{+}\right)$and all $\kappa \in[0, R]$,

$$
\begin{aligned}
\int_{0}^{+\infty} \int_{\mathbb{R}}\left(|\rho(t, x)-\kappa| \partial_{t}+\right. & \left.\Phi(\rho(t, x), \kappa) \partial_{x}\right) \phi(t, x) d x d t \\
& +\int_{\mathbb{R}}\left|\rho_{0}(x)-\kappa\right| \phi(0, x) d x+2 \int_{0}^{+\infty}(f(\kappa)-\min \{f(\kappa), q(t)\}) \phi(t, 0) d t \geq 0
\end{aligned}
$$

(ii) it verifies the constraint:

$$
f\left(\left(\gamma^{l} \rho\right)(t)\right)=f\left(\left(\gamma^{r} \rho\right)(t)\right) \leq q(t) \quad \text { a. e. } t>0 .
$$

Note that the last integrand in (21) differs from the corresponding term in (7). More precisely, condition (21) is finer, since

$$
f(\kappa)-\min \{f(\kappa), q(t)\} \leq\left(1-\frac{q(t)}{f_{\max }}\right) f(\kappa), \quad \text { for all } t>0, \kappa \in[0, R] .
$$


Moreover, condition (7) is not sufficient to rule out some non-maximal non-classical stationary shocks in the non-concave setting, see [4, Remark 2] for an example.

Definition 4.1 guarantees well posedness. In fact, an analogous of Proposition 2.3 and Theorem 2.4 is proved in [4]. Another application to pedestrian flows in treated in [1].

\subsection{The Aw-Rascle model with flux constraints}

The Aw-Rascle traffic flow model [3] belongs to the class of so-called "second order" models, i.e. models consisting in two equations. It can be written (in conservative form)

$$
\left\{\begin{array}{l}
\partial_{t} \rho+\partial_{x}(\rho v)=0 \\
\partial_{t}(\rho(v+p(\rho)))+\partial_{t}(\rho v(v+p(\rho)))=0
\end{array}\right.
$$

where $\rho$ and $v$ denote the density and mean speed of cars, respectively, and $p \in C^{2}([0,+\infty[;[0,+\infty[)$ is a pressure function satisfying the following hypotheses

$$
\begin{aligned}
& p(0)=0, \\
& p^{\prime}(\rho)>0 \text { for every } \rho>0, \\
& \rho \mapsto \rho p(\rho) \text { is strictly convex. }
\end{aligned}
$$

The first equation in (23) states the conservation of the number of vehicles, moving with flow rate $\rho v$. The second equation is derived from the former one and from the evolution equation of the quantity $w=v+p(\rho)$ (often referred to as "Lagrangian marker"), which moves with velocity $v$ :

$$
\partial_{t}(v+p(\rho))+v \partial_{x}(v+p(\rho))=0 .
$$

System (23) belongs to the Temple class [15], i.e. systems for which shock and rarefaction curves in the unknowns' space coincide. In particular, for such systems the interaction of two waves of the same family only gives rise to a wave of the same family.

In [11], we studied the Riemann problem

$$
\left\{\begin{array}{l}
\partial_{t} \rho+\partial_{x}(\rho v)=0 \\
\partial_{t}(\rho(v+p(\rho)))+\partial_{x}(\rho v(v+p(\rho)))=0 \\
(\rho, v)(0, x)= \begin{cases}\left(\rho^{l}, v^{l}\right), & \text { if } x<0 \\
\left(\rho^{r}, v^{r}\right), & \text { if } x>0\end{cases}
\end{array}\right.
$$

under the constraint

where $q \in \mathbb{R}^{+}$is a given constant.

$$
\rho(t, 0) v(t, 0) \leq q
$$

For any $\left(\rho^{l}, v^{l}\right) \in \mathbb{R}^{+} \times \mathbb{R}^{+}$and $q>0$, let us consider the set

$$
I_{1}=\left\{\rho \in \left[0,+\infty\left[: \rho\left(v^{l}+p\left(\rho^{l}\right)-p(\rho)\right)=q\right\} .\right.\right.
$$

The set $I_{1}$ contains the densities of all the points $(\rho, v) \in \mathbb{R}^{+} \times \mathbb{R}^{+}$belonging to the Lax curve of the first family passing through $\left(\rho^{l}, v^{l}\right)$ and such that $\rho v=q$. If $I_{1} \neq \emptyset$, then we denote by $\hat{\rho}, \hat{v}, \check{\rho}_{1}, \check{v}_{1}$ respectively

$$
\hat{\rho}=\max I_{1}, \quad \hat{v}=\frac{q}{\hat{\rho}}, \quad \check{\rho}_{1}=\min I_{1}, \quad \check{v}_{1}=\frac{q}{\check{\rho}_{1}} .
$$

Given $\left(\rho^{r}, v^{r}\right) \in \mathbb{R}^{+} \times \mathbb{R}^{+}$and $q>0$, let $\check{\rho}_{2}$ and $\check{v}_{2}$ be defined by

$$
\check{\rho}_{2} v^{r}=q, \quad \check{v}_{2}=\frac{q}{\check{\rho}_{2}} ;
$$


i.e. $\left(\check{\rho}_{2}, \breve{v}_{2}\right)$ belongs to the Lax curve of the second family passing through $\left(\rho^{r}, v^{r}\right)$ and satisfies $\check{\rho}_{2} \check{v}_{2}=q$. In particular, note that $\check{v}_{2}=v^{r}$ and $\check{\rho}_{2}=q / v^{r}$.

Two Riemann solvers are proposed: the first one conserves both the number of cars and the generalized momentum at $x=0$, while the second one does not conserve the generalized momentum. In particular, the first Riemann solver produces a non-entropic shock wave at $x=0$, which travels with zero velocity. Let $\mathcal{R S}: \mathbb{R}^{+} \times \mathbb{R}^{+} \times \mathbb{R}^{+} \times \mathbb{R}^{+} \rightarrow \mathbf{L}^{\mathbf{1}}\left(\mathbb{R} ; \mathbb{R}^{+} \times \mathbb{R}^{+}\right)$denote the classical Riemann solver for (25). Also, we denote the first component of the flux by $f_{1}(\rho, v):=\rho v$.

Definition 4.2. The Riemann solver $\mathcal{R} \mathcal{S}_{1}^{q}$ is defined as follows.

- If $f_{1}\left(\mathcal{R S}\left(\left(\rho^{l}, v^{l}\right),\left(\rho^{r}, v^{r}\right)\right)(0)\right) \leq q$, then

$$
\mathcal{R} \mathcal{S}_{1}^{q}\left(\left(\rho^{l}, v^{l}\right),\left(\rho^{r}, v^{r}\right)\right)(\lambda)=\mathcal{R S}\left(\left(\rho^{l}, v^{l}\right),\left(\rho^{r}, v^{r}\right)\right)(\lambda)
$$

for every $\lambda \in \mathbb{R}$.

- If $f_{1}\left(\mathcal{R S}\left(\left(\rho^{l}, v^{l}\right),\left(\rho^{r}, v^{r}\right)\right)(0)\right)>q$, then

$$
\mathcal{R S}_{1}^{q}\left(\left(\rho^{l}, v^{l}\right),\left(\rho^{r}, v^{r}\right)\right)(\lambda)= \begin{cases}\mathcal{R S}\left(\left(\rho^{l}, v^{l}\right),(\hat{\rho}, \hat{v})\right)(\lambda), & \text { if } \lambda<0 \\ \mathcal{R S}\left(\left(\check{\rho}_{1}, \check{v}_{1}\right),\left(\rho^{r}, v^{r}\right)\right)(\lambda), & \text { if } \lambda>0\end{cases}
$$

Definition 4.3. The Riemann solver $\mathcal{R} \mathcal{S}_{2}^{q}$ is defined as follows.

- If $f_{1}\left(\mathcal{R S}\left(\left(\rho^{l}, v^{l}\right),\left(\rho^{r}, v^{r}\right)\right)(0)\right) \leq q$, then

$$
\mathcal{R S}_{2}^{q}\left(\left(\rho^{l}, v^{l}\right),\left(\rho^{r}, v^{r}\right)\right)(\lambda)=\mathcal{R} \mathcal{S}\left(\left(\rho^{l}, v^{l}\right),\left(\rho^{r}, v^{r}\right)\right)(\lambda)
$$

for every $\lambda \in \mathbb{R}$.

- If $f_{1}\left(\mathcal{R S}\left(\left(\rho^{l}, v^{l}\right),\left(\rho^{r}, v^{r}\right)\right)(0)\right)>q$, then

$$
\mathcal{R S}_{2}^{q}\left(\left(\rho^{l}, v^{l}\right),\left(\rho^{r}, v^{r}\right)\right)(\lambda)= \begin{cases}\mathcal{R S}\left(\left(\rho^{l}, v^{l}\right),(\hat{\rho}, \hat{v})\right)(\lambda), & \text { if } \lambda<0 \\ \mathcal{R S}\left(\left(\check{\rho}_{2}, \check{v}_{2}\right),\left(\rho^{r}, v^{r}\right)\right)(\lambda), & \text { if } \lambda>0 .\end{cases}
$$

Several properties of the above Riemann solvers were studied in [11]. In particular, $\mathcal{R S}_{1}^{q}$ satisfies a maximum principle property on the second Riemann invariant $w=v+p(\rho)$, while $\mathcal{R S}_{2}^{q}$ induces a lower increase in the total variation of the density $\rho$, the generalized momentum $\rho(v+p(\rho))$ and the velocity $v$. Also, finite volume numerical schemes derived from Godunov's were designed both for $\mathcal{R S}_{1}^{q}$ and $\mathcal{R} \mathcal{S}_{2}^{q}$.

\subsection{Moving bottlenecks}

The presence of slow large vehicle (e.g. a bus) that reduces the road capacity and generates a moving bottleneck for the surrounding traffic flow can be modeled with a PDE-ODE coupled system consisting in a scalar conservation law representing the traffic flow with a density constraint at the bus position and an ODE describing the slower vehicle trajectory:

$$
\begin{cases}\partial_{t} \rho+\partial_{x} f(\rho)=0, & x \in \mathbb{R}^{+}, t>0 \\ \rho(0, x)=\rho_{0}(x), & x \in \mathbb{R} \\ \rho(t, y(t)) \leq \alpha R, & t>0 \\ \dot{y}(t)=\omega(\rho(t, y(t)+)), & t>0 \\ y(0)=y_{0} & \end{cases}
$$

Above $\rho=\rho(t, x) \in\left[0, \rho_{\max }\right]$ is the scalar conserved quantity denoting the mean traffic density and $R$ is the maximal density allowed on the road. The flux function $f(\rho):[0, R] \rightarrow \mathbb{R}^{+}$is given by the usual flux-density 
relation $f(\rho)=\rho v(\rho)$, where $v$ is set to be $v(\rho)=V\left(1-\frac{\rho}{R}\right)$, with $V$ the maximal velocity allowed on the road. The function $y=y(t)$ represents the slower vehicle position, which moves with a speed

$$
\omega(\rho)= \begin{cases}V_{b} & \text { if } \rho \leq \rho^{*} \doteq R\left(1-\frac{V_{b}}{V}\right) \\ v(\rho) & \text { otherwise }\end{cases}
$$

Hence, the slower vehicle moves with a constant speed $V_{b}<V$ as long as it is not slowed down by the downstream traffic conditions. At this point it will move at the same speed of the main traffic.

The coefficient $\alpha \in] 0,1$ [ gives the reduction rate of the road capacity due to the presence of the large vehicle. Notice that the density constraint in (26) is equivalent to the corresponding constraint on the flux

$$
f(\rho(t, y(t)))-\dot{y}(t) \rho(t, y(t)) \leq f_{\alpha}\left(\rho_{\alpha}\right)-\dot{y}(t) \rho_{\alpha} \doteq F_{\alpha}
$$

where $f_{\alpha}(\rho)=\rho\left(1-\frac{\rho}{\alpha}\right)$ and $\rho_{\alpha}=\frac{\alpha}{2}\left(1-V_{b}\right)$. When the constraint is active, the bus velocity satisfies $\dot{y}(t)=V_{b}$.

The definition of the corresponding weak solutions and Riemann solver, as well an existence result are provided in [8]. A finite volume numerical method is also proposed in [9]. (More details are provided in another contribution of the same volume.)

\section{REFERENCES}

[1] B. Andreianov, C. Donadello, and M. D. Rosini. Crowd dynamics and conservation laws with non-local constraints. Preprint, Apr. 2013.

[2] B. Andreianov, P. Goatin, and N. Seguin. Finite volume schemes for locally constrained conservation laws. Numer. Math., 115(4):609-645, 2010. With supplementary material available online.

[3] A. Aw and M. Rascle. Resurrection of "second order" models of traffic flow. SIAM J. Appl. Math., 60(3):916-938 (electronic), 2000.

[4] C. Chalons, P. Goatin, and N. Seguin. General constrained conservation laws. Application to pedestrian flow modeling. Netw. Heterog. Media, 8(2):433-463, 2013.

[5] R. M. Colombo and P. Goatin. A well posed conservation law with a variable unilateral constraint. J. Differential Equations, 234(2):654-675, 2007.

[6] R. M. Colombo, P. Goatin, and M. D. Rosini. On the modelling and management of traffic. ESAIM Math. Model. Numer. Anal., 45(5):853-872, 2011.

[7] R. M. Colombo and M. D. Rosini. Pedestrian flows and non-classical shocks. Math. Methods Appl. Sci., 28(13):1553-1567, 2005.

[8] M. L. Delle Monache and P. Goatin. Scalar conservation laws with moving density constraints arising in traffic flow modeling. Rapport de recherche RR-8119, INRIA, Oct. 2012.

[9] M. L. Delle Monache and P. Goatin. A front traking method for a strongly coupled PDE-ODE system with moving density constraints in traffic flow. Discrete Contin. Dyn. Syst. Ser. S, to appear.

[10] R. Eymard, T. Gallouët, and R. Herbin. Finite volume methods. In Handbook of numerical analysis, Vol. VII, Handb. Numer. Anal., VII, pages 713-1020. North-Holland, Amsterdam, 2000.

[11] M. Garavello and P. Goatin. The Aw-Rascle traffic model with locally constrained flow. J. Math. Anal. Appl., 378(2):634-648, 2011.

[12] K. H. Karlsen and J. D. Towers. Convergence of the Lax-Friedrichs scheme and stability for conservation laws with a discontinous space-time dependent flux. Chinese Ann. Math. Ser. B, 25(3):287-318, 2004.

[13] S. N. Kružhkov. First order quasilinear equations with several independent variables. Mat. Sb. (N.S.), 81 (123):228-255, 1970.

[14] E. Y. Panov. Existence of strong traces for quasi-solutions of multidimensional conservation laws. J. Hyperbolic Differ. Equ., $4(4): 729-770,2007$.

[15] B. Temple. Systems of conservation laws with coinciding shock and rarefaction curves. Contemp. Math., 17:143-151, 1983.

[16] A. Vasseur. Strong traces for solutions of multidimensional scalar conservation laws. Arch. Ration. Mech. Anal., 160(3):181-193, 2001. 